\title{
Polynomial Root Solving on the Electronic Differential Analyser (A Technique for Finding the Real and Complex Roots of a Polynomial using an Electronic Differential Analyser)
}

1. Introduction. This paper presents a technique for the solution of high degree polynomials with real coefficients. By means of this technique the real and complex roots of the polynomial

$$
f(x)=a_{0} x^{n}+a_{1} x^{n-1}+\cdots+a_{n} x^{2}
$$

can be obtained. The technique is essentially a graphic one in which the function $f(x)$ and its derivatives $f^{\prime}(x), f^{\prime \prime}(x), \cdots$ are generated by an electronic differential analyser [1]. The method depends heavily on the ability of the analyser to produce an accurate plot of the function and its derivatives. The use of these accurate graphs is obvious for finding the real roots of the equation. But a systematic use of graphs for the finding of complex roots is not so obvious and hence not widely used [2].

This computer approach to the finding of the complex roots requires additional curves of the real and complex parts of the complex function

$$
U+i V=f(z)=a_{0} z^{n}+a_{1} z^{n-1}+\cdots+a_{n} ; \quad(z=x+i y) .
$$

The real part $U(x, y)$ and the complex part $V(x, y)$ are also plotted by the analyser and complex roots are found from the common zeros.

2. Method of Solution.

1. Real Roots. In order to generate the equation $y=f(x)$ on the differential analyser the polynomial must be differentiated $n$ times, where $n$ is the degree of the polynomial. This will produce the derivatives $f^{n}(x), f^{n-1}(x), \cdots, f^{\prime}(x)$ where $f^{n}(x)$ is a constant, say $f^{n}$. If a voltage of value $f^{n}$ is introduced into an integrating amplifier the output is $x f^{n}$. (See Fig. 1a.) Putting the value of $f^{n-1}(0)$ into the same integrator makes the output $f^{n-1}(x)$ (neglecting the sign change of the amplifier). This process is continued until the final integration produces $f(x)$. The real roots are picked from the curve as the values of $x$ for which $f(x)=0$.

2. Complex Roots. The technique for finding the complex roots of the polynomial is based on the idea of a surface $f(z)$ over the horizontal $x y$ plane where

$$
f(z)=f(x+i y)=U(x, y)+i V(x, y) .
$$

For $f(z)$ real, $V(x, y)=0 ; f(z)=U(x, y)$ so $U(x, y)$ is a space curve whose projection on the $x y$ plane is $V(x, y)=0$.

For the solution of the polynomial equation $f(z)=U+i V=0$ both the real function $U$ and the imaginary function $V$ must be zero so the root $x_{1}+i y_{1}$ represents the intersection of the space curve $U(x, y)$ with the curve $V(x, y)=0$. This is a point in the $x y$ plane where the $U$ function is zero. 

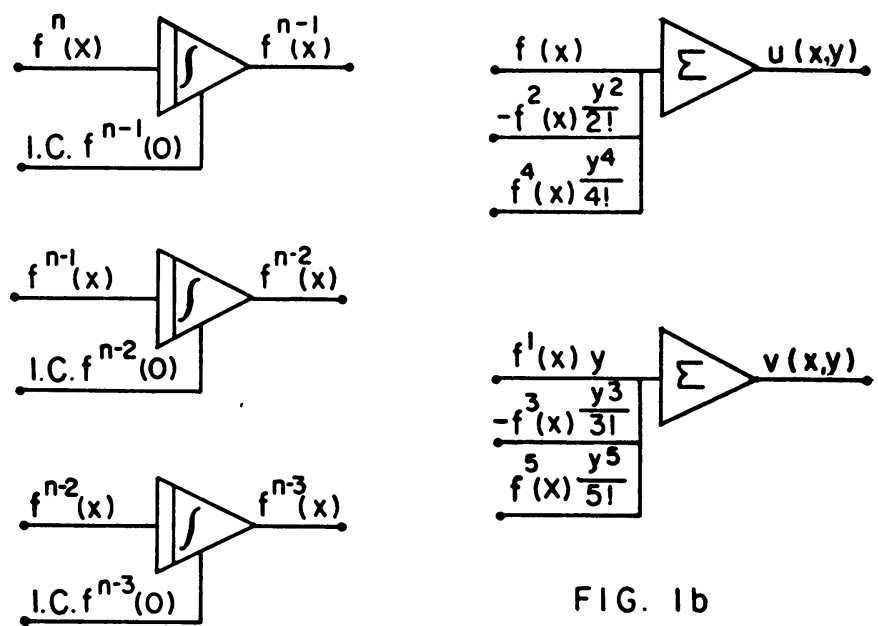

FIG. I b

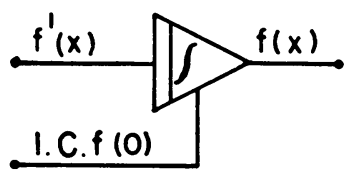

LEGEND

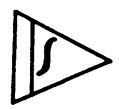

INTEGRATOR

FIG. 10

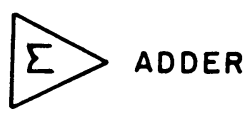

\section{I.C. INITIAL CONDITION}

FIG. 1. General wiring diagram for polynomial root solving. Sign inverters have not been shown.

The differential analyser can handle only one variable of integration so the common point in the $x y$ plane where both $U$ and $V$ are zero must be approached by a judicious selection of one of the variables, usually $y=y_{1}$. Then a plot of the two functions $U\left(x, y_{1}\right)$ and $V\left(x, y_{1}\right)$ reveals how close $y_{1}$ is to the actual imaginary part of the root. If the plot of $U_{1}$ and $V_{1}$ indicates a common crossing of the axis, say at $x_{1}$, then the chosen $y_{1}$ is the correct one and the root is $x_{1}+i y_{1}$. Notice that this also yields the conjugate root $x_{1}-i y_{1}$.

If the curves $U_{1}$ and $V_{1}$ do not have a common crossing of the abscissa, a change in $y_{1}$ is made and another plot of the two equations is generated. This is continued until the root is obtained to the desired accuracy.

The judicious choice of $y_{1}$ is assisted by a knowledge of the positions of maxima, minima, points of inflection, and accessory points of inflection (where a maximum and a minimum have run together) of the real curve.

A. J. KEMPNER's [2] suggestion that a study of the derivatives is helpful in estimating the complex roots of a polynomial is borne out by the nature of the real and imaginary parts of the complex function $f(z)$. The real part

$$
U(x, y)=f(x)-f^{\prime \prime}(x) y^{2} / 2 !+f^{(\mathrm{IV})}(x) y^{4} / 4 !-\cdots,
$$




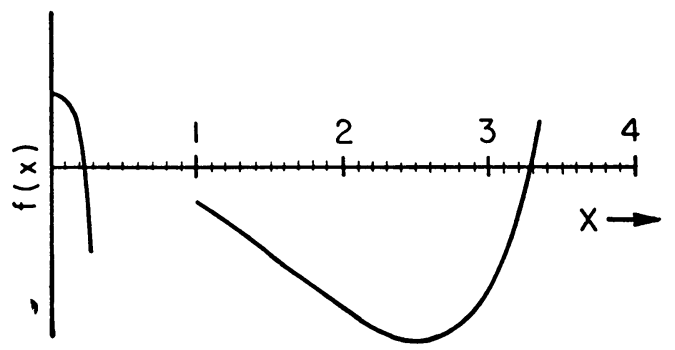

FIG. 2. Graph showing real roots of quintic equation $x^{5}+x^{4}-7 x^{3}-22 x^{2}+x+1=0$ of example 1. Note: Change of vertical scale at $X=1$.

and the imaginary part

$$
V(x, y)=f^{\prime}(x) y-f^{\prime \prime \prime}(x) y^{3} / 3 !+f^{(\mathrm{V})}(x) y^{5} / 5 !-\cdots ;
$$

that is, the real part is the alternating sum of the polynomial and its even derivatives multiplied by the corresponding even powers of $y$ and inverse factorials, while the imaginary part is the sum of the odd derivatives multiplied by the odd powers of $y$ and corresponding inverse factorials.

Since the respective derivatives are generated in order to produce the function $f(x)$ (as shown in Fig. 1a) they are available for finding a first approximation to the imaginary part of a complex root. They are then used for producing $U\left(x, y_{1}\right)$ and $V\left(x, y_{1}\right)$ after being multiplied by the appropriate factor (e.g., $f^{\prime \prime}(x)$ is multiplied by $y_{1}^{2} / 2$ !). (See Fig. 1b.)

\section{Examples of Computer Solutions.}

Example 1. Real Roots of a Quintic Equation. The polynomial

$$
x^{5}+x^{4}-7 x^{3}-22 x^{2}+x+1=f(x)
$$

is used as an example for the machine solution of real roots. The roots sought are the real positive roots [3]. This requires only the generation of the respective derivatives (Fig. 1a) to produce $f(x)$. The computer plotted the function as shown in Fig. 2. The curve crossed the $x$ axis at the roots 0.22 and 3.28.

Example 2. Complex Roots of a Quartic Equation. The polynomial

$$
x^{4}+x^{3}+5 x^{2}+5 x+12=f(x)
$$

is used as an example for the machine solution of complex roots [4]. The computer

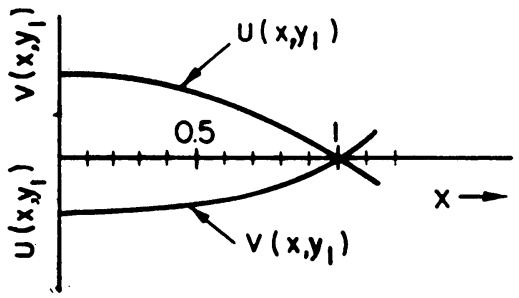

FIG. 3. Graph showing common intersection of $x$ axis by $U\left(x, y_{1}\right)$ and $V\left(x, y_{1}\right)$ indicating the roots $-1 \pm i \sqrt{2}$ of the equation $x^{4}+x^{3}+5 x^{2}+5 x+12=0$. 
is wired as in Fig. 1a and Fig. $1 \mathrm{~b}$ and $U$ and $V$ curves are plotted for negative $x$ with $y=\sqrt{2}$. Fig. 3 indicated the simultaneous crossing of the axis at $x=1$. Since the computer plotted the negative halves of the curves this crossing is then the conjugate pair $-1 \pm i \sqrt{2}$.

Example 3. Sixth Order Polynomial with Three Pairs of Complex Roots. The polynomial [5]

$$
x^{6}-x^{5}+2 x^{4}-3 x^{3}+2 x^{2}+x+1=f(x)
$$

was chosen as a fairly difficult equation to solve by this method since all roots are complex and clustered about the origin.

Figure 4 is a plot of the expressions $U$ and $V$ for the value of $y_{1}=0.64$. The pair of roots as given by the computer was $1.0 \pm 0.64 i$. (These roots are given in USPENSKY as $1.08018 \pm 0.63903 i$.)

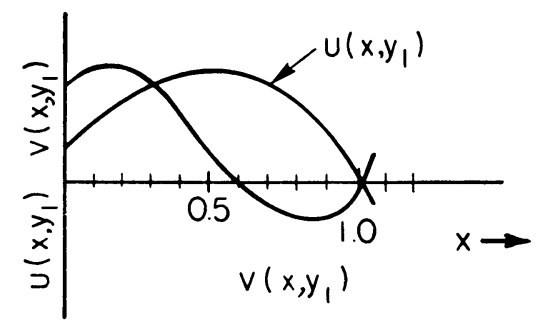

FIG. 4. Graph showing common intersection of $x$ axis by $U\left(x, y_{1}\right)$ and $V\left(x, y_{1}\right)$ indicating the roots $1.08 \pm 0.64 i$ of the equation $x^{6}-x^{5}+2 x^{4}-3 x^{3}+2 x^{2}+x+1=0$.

4. Conclusion. The method of root solving presented herein is a new technique employing the latest type of analog computer to the old problem of root solving. The solutions generated by the computer are fast as compared with the computational methods or the mechanical root finders. The accuracy of roots can easily be kept in the range of engineering accuracy. Mathematical manipulations of the original equation are minimized by the method which requires only the derivatives of the polynomial whose roots must be determined.

University of Calif.

Cyril Atkinson

Berkeley, Calif.

1. The electronic differential analyser used is the Beckman EASE computer located at the Timoshenko Laboratory, Stanford University.

2. A. J. KEMPNER, "On the Complex Roots of Algebraic Equations," Amer. Math. Soc., Bull., v. 41, 1935, p. 809-843.

3. J. V. Uspensky, Theory of Equations, McGraw-Hill, N. Y., 1948.

4. See reference 3 for roots $-1 \pm i \sqrt{2} ; \frac{1}{2} \pm \frac{i \sqrt{15}}{2}$.

5. See reference 3 appendix V, p. 318-331, for the solution by GRAEFFE's method.

6. A. R. Boothroyd, E. C. CHerRy, \& R. MAKar, "An electrolytic tank for the measurement of steady-state response, transient response, and allied properties of networks," I.E.E., Proc., v. 96, Part I, 1949, p. 163-177.

7. R. L. Dietzold, “The Isograph-a mechanical root finder," Bell Laboratories Record, v. 16, 1937-38, p. 130.

8. G. FERrara \& R. L. Nadeau, “A complex wave synthesizer," Elec. Eng., v. 70, 1951, p. 585.

9. J. S. FRAME, "Machines for solving algebraic equations," $M T A C$, v. 1, 1945, p. 337-353.

10. H. C. HART \& I. TRAvIS, "Mechanical solution of algebraic equations," J. Franklin Inst., v. 215,1938, p. $63-72$. 
11. A. J. Kempner, "Geometry as an avocation," Amer. Math. Mon., v. 40, 1933, p. 455-471.

12. Byron O. MARshall, JR., "The electronic isograph for roots of polynomials," $J$. $A p p l$. Phys., v. 21, 1950, p. 307-312.

13. J. H. RoBERTSON, "A simple machine capable of Fourier synthesis calculation," J. Sci. Instr., v. 27, 1950, p. 276-278.

14. A. Russell \& J. N. Alty, "An electromagnetic method of studying the theory of and solving algebraic equations of any degree," Phil. Mag., s. 6, v. 18, 1909, p. 802-812.

15. T. B. RYMER \& C. C. BUTLER, "An electrical circuit for harmonic analysis and other calculations," Phil. Mag., s. 7, v. 35, 1944, p. 606-616.

16. Walter W. SorokA, Analog Methods in Computation and Simulation, McGraw-Hill, New York, 1954.

17. L. BAUER \& S. FIFER, "The solution of polynomial equations on the REAC," Project Cyclone Symposium One, Reeves Instrument Corp., New York, 1951, p. 31-36.

18. F. W. J. Olver, "The evaluation of zeros of high-degree polynomials," Royal Soc. of London, Phil. Trans., ser. A, v. 244, 1952, p. 385-415.

19. R. A. Brooker, "The solution of algebraic equations on the EDSAC," Cambridge Phil. Soc., Proc., v. 48, 1952, p. 255-270.

\section{Notations for Partitions}

The present paper gives a contribution to the automatic calculation of the characters of certain symmetric groups, as described in a paper which recently appeared in $M T A C$ [1]. The repeated application of the recursion formula (7) of the cited paper involves the storing of a chain of partitions. The allotted storage space must be sufficient to meet even the case of a chain of as many as about $n / 2$ partitions. In other words, provision must be made for storing about $n^{2} / 4$ integers, components of these partitions. It would be a waste of memory space to store these integers separated, numerous and small as they are. Furthermore, as $n$ increases, it will be still more important that the partitions should be available in the rapid access storage of the machine. Therefore, they should be stored by some method of packing, suitable for the operations to be applied, particularly during the calculation of characters.

In the sequel, I shall briefly describe three such methods, which might perhaps be of some interest. The first method [2] was used in an experimental program for character calculation with the Swedish relay computer BARK (August, 1953) and also in the first version of the character program using the Swedish electronic computer BESK (Spring, 1954). The second method is employed in the actual version of this program (tested on BESK, June, 1954). The third method, a modification of the second, has not yet been used in the program, though it is described here for theoretical reasons.

Preliminaries. Theoretically, the minimum of the number, $x$, of binary digits required to represent all the partitions of an integer $n$, is the smallest integer, not less than $\log _{2} P(n)$, where $P(n)$, the number of the partitions, can be estimated by means of HARDY-RAMANUJAN's formula [3]. The result obtained,

$$
x_{\text {min }} \sim \pi \sqrt{2 / 3} \log _{2} e \cdot \sqrt{n}-\log _{2} 4 n \sqrt{3} \sim 3.70 \sqrt{n}-\log _{2} n-2.79
$$

indicates an ideal towards which the packing methods should lead.

If packing with fixed boundaries between the packed elements, one should store the partition components, each diminished by 1 . In this case, $n$ must also be stored. The value of $x$ then lies between $n$ and $3 n / 2$. For example, in a 40 digit register one can store the partitions of $n \leq 30$ by this method. It will be seen that 